\title{
EL FACTOR “TIPO DE TEXTO” EN LA COMPRENSIÓN LECTORA DEL FRANCÉS LENGUA EXTRANJERA ${ }^{1}$
}

\author{
Leyre Ruiz de Zarobe \\ Universidad del País Vasco
}

\begin{abstract}
Resumen: El objeto del presente artículo es analizar la incidencia del factor "tipo de texto" en la comprensión lectora del Francés Lengua Extranjera, a la luz de las investigaciones recientes más relevantes en Psicolingüística. Siendo la lectura una competencia que los locutores tienen interiorizada en su lengua materna, nos hemos remitido en primer lugar a los estudios más destacados sobre la incidencia de la tipología textual en la lectura en Francés Lengua Materna, para después abordarlo en Francés Lengua Extranjera. Extraemos algunas conclusiones relativas al estado actual de esta cuestión, y también a los ejes por lo que, en nuestra opinión, es necesario progresar en la investigación.
\end{abstract}

Palabras clave: tipo de texto -comprensión lectora - francés lengua materna - francés lengua extranjera.

\begin{abstract}
The aim of the present article is to analyse the influence of the factor "text type" on reading comprehension in French as a Foreign Language, in the light of recent research in Psycholinguistics. Reading is a competence that subjects know in their own language; that is why we study first the influence of text type in French as a mother tongue, to analyse it then as a Foreign Language. We show some conclusions about the actual state of this subject, and also some paths through which, in our opinion, investigation should progress.
\end{abstract}

Keywords: text type - reading comprehension - French as a mother tongue - French as a foreign language.

\section{INTRODUCCIÓN}

La comprensión de los textos ligada a su tipología (descriptiva, narrativa, argumentativa...) constituye hoy en día un centro de interés en Lingüística Aplicada, y en Psicolingüística. La investigación en Psicolingüística muestra que uno de los fundamentos de la comprensión reside en el reconocimiento de los tipos textuales convencionales, y que los llamados esquemas textuales intervienen en la comprensión lectora de los textos. Más precisamente, como afirman Golder y Gaonac'h (1998: 93) « les schémas textuels constituent des outils cognitifs permettant de produire et de comprendre les textes ».

Los esquemas textuales (narración, argumentación, exposición, etc.) remiten básicamente a la forma y organización de los textos, y construyen los tipos de texto específicos. Generalmente, esos esquemas están convencionalizados en una cultura dada, y se adquieren por convención.

Algunos autores como Kintsch y Van Dijk (1983) hablaron del concepto de superestructura textual, concepto que puede ser asimilado al de esquema textual. La superestructura se define por los constituyentes que la forman, su orden, y su estructura jerárquica. Se espera que la superestructura intervenga en la comprensión de los textos atribuyendo información a cada

Este estudio se enmarca en nuestro Proyecto de Investigación HUM2004-05403/FILO, financiado por el MEC. 
elemento de la superestructura, o realizándolo en un orden determinado, o avanzando información, etc.

Así pues, se considera que los esquemas textuales, o las superestructuras textuales, o los tipos de texto (en la terminología de Adam), en tanto que reflejan regularidades, controlan la comprensión de los textos. Lo pertinente será mostrar la existencia de esos esquemas textuales cognitivos en los individuos, como veremos más adelante.

El objeto de este artículo es analizar la incidencia de la tipología textual en la comprensión lectora de una lengua concreta, el francés como lengua extranjera, a la luz de las investigaciones más relevantes, en este ámbito, en Psicolingüística.

Siendo la lectura una competencia que los locutores tienen interiorizada en su lengua materna, nos hemos remitido en primer lugar a los estudios más destacados sobre la incidencia de la tipología textual en la lectura en Francés Lengua Materna, para después abordarlo en Francés Lengua Extranjera, teniendo en cuenta las características esenciales de la lectura en lengua extranjera.

\section{TIPO DE TEXTO Y COMPRENSIÓN LECTORA EN FRANCÉS LENGUA MATERNA}

De manera genérica podemos decir que leer y comprender un texto constituye una actividad de reconstrucción de una representación mental de la situación, en la cual la significación no se extrae directamente del texto, sino que se construye a partir de los conocimientos previos del lector, y se integra en ellos.

Lire est par essence un processus dynamique, qui nécessite de la conduite simultanée de plusieurs activités. [...] II s'agit toujours de construire une représentation -une interprétation- qui respecte ce que l'auteur du texte a effectivement redigé, tout en faisant appel aux connaissances conceptuelles et langagières du lecteur, qui à la fois permettent et limitent son interprétation.

De ce point de vue, la compréhension réalisée au cours de la lecture, comme la compréhension en général, ne peut se satisfaire de la construction de représentations fragmentaires juxtaposées, sans organisation leur donnant unité et cohérence» (Gaonac'h y Fayol $2003: 9$ ).

Desde los años setenta, con el desarrollo de los estudios sobre el texto, se busca también aplicar los conocimientos sobre el texto al fenómeno de la comprensión de textos. En este sentido, a partir de dichos trabajos existe una preocupación en examinar la influencia de los distintos factores que constituyen el texto, en la actividad cognitiva de comprensión.

\subsection{Operaciones cognitivas ligadas a la comprensión de los tipos de texto}

La hipótesis de partida en la investigación sobre la comprensión de la tipología textual es la creencia de que los lectores poseen una capacidad de reconocer un texto como narrativo, descriptivo, argumentativo, etc. 
La cuestión que surge a continuación es el planteamiento de si los textos pertenecientes a un mismo tipo, aunque de contenido diferente, serán tratados por el lector de la misma manera, $y$, correlativamente, si los textos pertenecientes a tipos diferentes, aunque con el mismo contenido, se tratarán de manera diferente. Ambos aspectos parecen tener una respuesta positiva, de acuerdo con los estudios de Fayol (1991).

La Psicolingüística se plantea así que los diferentes tipos de texto llevan a pensar en esquemas cognitivos potenciales de tratamiento para cada tipo textual. De hecho, el que un texto pertenezca a un tipo u otro puede influir en determinadas operaciones cognitivas (Coirier y alt. 1996). ¿Cuáles son esas operaciones?

En primer lugar, un tipo de texto puede activar su esquema superestructural (organización convencional), y por tanto, la jerarquización y organización de la información textual. Por ejemplo, leer un texto narrativo puede activar su esquema superestructural, que sería: marco-temacomplicación-resolución-evaluación y lección. La lectura de un texto argumentativo interviene en la activación de un esquema como tesis-argumentos-conclusión.

En segundo lugar, un tipo de texto puede influir en la activación no sólo de un esquema textual, sino de un esquema cognitivo más general, como puede ser la causalidad en el texto narrativo, las estructuras lógicas en el texto científico, etc.

En tercer lugar, el tipo de texto puede poner de manifiesto ciertos dominios de conocimiento, como el dominio de referencia. Por ejemplo, un texto narrativo se basa en hechos que se suceden en un orden temporal y causal. Una argumentación se basa en una relación de argumentos, si no preestablecidos, sí construidos por el lector. Un texto expositivo puede basarse en una explicación, una analogía, etc.

En cuarto lugar, leer un tipo de texto puede llamar a la activación de marcas lingüísticas específicas, como los localizadores espaciales en los textos descriptivos, o los conectores argumentativos en el texto argumentativo...

Esta es una muestra de las operaciones cognitivas en las que puede intervenir un tipo de texto particular, pero sin duda, existen más.

Lo que resulta evidente es que los distintos tipos de textos no se distinguen sólo por una dimensión, sino por varias, $y$, por lo tanto, por estructuras cognitivas asociadas a tales dimensiones.

De todos estos elementos que configuran el texto, cabe plantearse si existe alguno más pertinente en el tratamiento de los textos, o, como parece, es la interacción de todos ellos lo que determina la lectura y comprensión de los textos.

\section{2. ¿Qué tipos de texto?}

Numerosas han sido las tipologías que se han propuesto para clasificar los textos, y desde criterios muy diversos, como funcional, enunciativo, sociológico... tipologías de las que no vamos a dar cuenta aquí. 
Las más pertinentes a nuestro propósito son las tipologías basadas en las estructuras cognitivas que se ponen en juego en los distintos tipos de texto. En este sentido, destacamos las de Werlich, Brewer, y Adam.

Werlich (1975), a cuya tipología se ha recurrido abundantemente en Psicolingüística, distingue cinco tipos de texto:

- el narrativo, ligado a la percepción del tiempo

- el descriptivo, ligado a la percepción del espacio

- el expositivo, ligado al análisis y síntesis de representaciones conceptuales

- el argumentativo, ligado a las posiciones y los juicios

- el instructivo, ligado a las previsiones

La propuesta de Werlich combina la perspectiva cognitiva (forma de concebir la realidad), con la lingüística (forma de representar la realidad).

Brewer (1980) propone una clasificación de los textos de acuerdo con dos dimensiones:

a) cognitiva. Cada estructura textual implica, para su tratamiento, a una actividad cognitiva.

De acuerdo con la dimensión cognitiva, el autor distingue tres tipos básicos de textos:

- el descriptivo, asociado a la percepción de las escenas estáticas

- el narrativo, asociado a hechos que se desarrollan en el tiempo, y ligados por cadenas temáticas o causales

- el expositivo/explicativo, donde intervienen procesos lógicos, como la inducción, comparación, etc.

b) ilocucionaria. Cada estructura textual posee una fuerza ilocucionaria, en el sentido de la teoría de los actos de habla. Así pues, los textos pueden tener distintas funciones: informar, distraer, convencer...

De alguna manera, se ligan, en la propuesta de Brewer, planteamientos de psicología cognitiva con planteamientos de pragmática textual.

Combinadas ambas dimensiones, se producen doce categorías potenciales de textos.

Brewer también defiende, por otro lado, que los textos no son homogéneos desde el punto de vista tipológico, sino heterogéneos. Es también el punto de vista de Adam. Adam propone no tipologías textuales a nivel de los textos generales, sino secuencias elementales, homogéneas. El texto sería un conjunto de secuencias, encadenadas las unas en las otras, o insertadas las unas en las otras.

La teoría de Adam en lo relativo a la tipología textual está hoy ampliamente reconocida, y figura como una de las teorías de referencia más relevantes sobre lingüística textual. Dicha teoría se encuadra en la prolongación de la teoría psico-cognitiva de los esquemas desarrollada 
principalmente por Kintsch y Van Dijk (1983). En ella, se asigna un sentido y un valor ilocutorio a las proposiciones; luego, esas proposiciones se procesan en bloque para ser almacenadas en la memoria de trabajo para seguir procesando sentido del texto, y por último se establece el reconocimiento de organizaciones convencionales que Kintsch y Van Dijk llaman superestructuras. Estos autores hablan de superestructuras refiriéndose tanto a la argumentación o a la narración, como a un artículo científico, o a un soneto. Adam precisará más la naturaleza de esos tipos dispares de unidades textuales, distinguiendo la secuencia y la estructura (plan) de texto.

Adam (1992, 1999, 2005) va a considerar el texto como Adam (1992: 21) "une configuration réglée par divers modules ou sous-systèmes en constante interaction". Tres módulos de organización pragmática :

- la visée ilocucionaria: todo texto tiene una función, explícita o no, que busca actuar sobre las creencias o conductas del destinatario.

- las referencias enunciativas: todo texto constituye un acto de enunciación por parte de un enunciador, en un tiempo y lugar determinado.

- la dimensión semántica global: todo texto está representado por la macroestructura semántica o el tema global.

Y dos módulos de organización de las proposiciones:

- la conexidad textual: todo texto está compuesto de proposiciones y períodos articulados entre sí.

- la organización secuencial: todo texto está compuesto de uno o varios esquemas secuenciales prototípicos.

Esta idea de secuencia es la más interesante para nosotros aquí. Como Adam dice, tanto en comprensión como en producción, parece que los sujetos van adquiriendo esas secuencias a lo largo de su desarrollo cognitivo.

Chaque séquence reconnue comme descriptive, par exemple, partage avec les autres un certain nombre de caractéristiques linguistiques d'ensemble, un air de famille qui incite le lecteur interprétant à les identifier comme des séquences descriptives plus ou moins typiques, plus ou moins canoniques. II en va exactement de même pour une séquence narrative, explicative ou argumentative » (Adam 1992 : 28).

En dicha obra, y también en su última versión de la teoría, Adam (2005) define la secuencia como una estructura, esto es, como una red relacional jerárquica (que se puede descomponer en partes ligadas entre sí), y como una entidad relativamente autónoma, con su propia organización interna.

Adam define cinco secuencias, memorizadas culturalmente, bien por la lectura, o la escucha, o la producción textual: 
- narrativa

- argumentativa

- explicativa

- dialogal

- descriptiva

Estas secuencias se convierten en esquemas que permiten estructurar la información textual, y también, ser reconocidos, como vemos en el punto siguiente.

\subsection{Identificación de los distintos tipos de texto}

En cuanto a la investigación relativa a la capacidad de los sujetos de reconocer los distintos tipos de texto, podemos decir que existen pocas investigaciones empíricas para la lengua francesa, con excepción del tipo narrativo.

Como hemos dicho más arriba, parece que la comprensión se fundamenta en la identificación o reconocimiento de los tipos de texto convencionales. Si tomamos, por ejemplo, el caso de la narración, la narración posee una estructura determinada, que, además, es compartida por muchas culturas: exposición, complicación, resolución, evaluación, lección. Se trataría de saber, si desde el punto de vista psicológico, existe esta misma estructura narrativa, que permitiría reconocer y tratar una narración, al leerla, u oírla.

Algunas investigaciones (Denhière 1984 ; Fayol 1985 ; Fayol 1996 ; Fayol 2000) han mostrado efectivamente la existencia de un esquema narrativo (exposición, complicación, resolución, evaluación, lección) que determina la comprensión, es decir, que permite integrar la información textual, tratando los textos relativamente a esas categorías. En estos estudios se muestra, además, que las narraciones que siguen un orden canónico se comprenden y se recuerdan mejor, o dicho de otra manera, la modificación del orden canónico de la narración hace que las narraciones se recuerden peor. Cuando no se lee la organización narrativa convencional, en el recuerdo se omiten informaciones o se restablece el orden canónico.

En cuanto al texto argumentativo, la existencia de un esquema no es tan evidente, probablemente por su organización no tan canónica como la del texto narrativo. Las investigaciones en Francés Lengua Materna como las de Coirier y Marchand (1994), Golder y Coirier (1994), Golder y Coirier (1996) muestran la existencia de un esquema argumentativo : el criterio de identificación del texto argumentativo es la estructura "posición-justificación », y desde los 12 años. Es decir, identificar esa estructura mínima es lo que permite reconoce un texto como argumentativo. Para otros autores como Brassart (1996), la existencia del esquema argumentativo no es tan clara:

If indeed there is an argumentative text schema, it is so impoverished that it is worth inquiring in what ways it facilitates processing (by concept) of argumentative texts in individuals who have mastered this schema" (Brassart 1996: 171).

Algo similar al texto argumentativo le sucede al texto explicativo, donde la estructura no está tampoco tan definida desde el punto de vista del contenido, y es difícil hablar de una estructura canónica. Así lo avanza Meyer (1975), que estudia la organización retórica variada 
de los textos explicativos: problema-solución, colección, causalidad, etc. Ahora bien, si se familiariza a los sujetos con dichas organizaciones, se desarrolla en ellos la capacidad de identificarlas correctamente, y de reconocerlas como propias del texto expositivo.

Los estudios de Meyer sobre las relaciones retóricas de los textos explicativos ha servido de base para estudios posteriores en comprensión lectora, como los de Carrell, por ejemplo.

\section{TIPO DE TEXTO Y COMPRENSIÓN LECTORA EN FRANCÉS LENGUA EXTRANJERA}

\subsection{Características esenciales de la lectura en lengua extranjera}

Cuando se aborda la lectura en una lengua extranjera, esta habilidad ya está adquirida en lengua materna. La lectura se compone de una serie de competencias que, de entrada, parece que se transfieren automáticamente a la lengua extranjera. Sin embargo, la primera dificultad con la que nos encontramos es la de la nueva grafía en lengua extranjera; si la grafía no es muy diferente a la de la lengua materna, las dificultades serán obviamente menores que si lo son, como puede ser el chino o japonés para un europeo.

La lectura es un actividad compleja en la que intervienen competencias llamadas de bajo nivel (descodificación gráfica, acceso léxico, estructuras sintáctico-semánticas), y de alto nivel (contexto, esquemas textuales); estas competencias de alto nivel son las que propiamente proporcionan el significado de un texto. La comprensión en la lectura se establece por esa interacción permanente entre las competencias de bajo y de alto nivel.

De entrada, cuando se aborda la lectura en lengua extranjera parece que las competencias que requieren un mayor grado de aprendizaje son las competencias de bajo nivel, al tratarse de un código lingüístico nuevo. Las competencias de alto nivel parece que pueden transferirse más directamente, especialmente si los rasgos sociolingüísticos y pragmáticos de las dos lenguas no son tan diferentes; si lo son, en tanto que desconocidas o nuevas, suponen un aprendizaje más para los estudiantes.

Sin embargo, la psicología cognitiva (Gaonac'h, 2000; Gaonac'h, y Fayol, 2003) muestra que lo que sucede en el aprendizaje de una lengua extranjera es que el acceso a dichas competencias queda bloqueado por un procesamiento insuficiente de las competencias de bajo nivel. Las competencias de bajo nivel, aunque conocidas, no se encuentran suficientemente automatizadas en el lector extranjero, el coste cognitivo es mayor, y eso dificulta la operatividad de los procedimientos de comprensión de alto nivel, y por tanto, la comprensión del texto en su globalidad. Todo esto tiene como consecuencia que se necesita mayor tiempo para comprender, o volver sobre el propio texto más veces, al saturarse el tratamiento de las distintas capacidades cognitivas que intervienen en la comprensión.

\subsection{La tipología textual en la lectura en Francés Lengua Extranjera}

Cuando se estudia la influencia de la tipología textual en la lectura, es obligado mencionar los estudios de Carrell, una importante especialista en el ámbito de la lectura en lengua extranjera. 
Carrell (1984), en uno de sus estudios, desea medir cómo los estudiantes recuerdan textos con organización estructural diferente: Collection, Description, Causation, Problem-Solution, y Comparison. Las estructuras que mejor se recuerdan son las más cohesionadas (Comparison, Causation, Problem-Solution). Los alumnos que reconocieron el tipo de estructura y la utilizaron en sus resúmenes recordaron mayor número de ideas. Es decir, si se posee el esquema para procesar el tipo de texto, éste se recuerda mejor.

En un estudio de Carrell (1990) con adultos que aprenden el Inglés Lengua Extranjera, se compara un relato bajo dos formas: una, un relato con una estructura canónica clara (exposición, complicación, resolución, conclusión), y otro, semánticamente igual, pero en el que el orden de los elementos se altera deliberadamente. Los resultados muestran que los relatos desordenados se recuerdan peor que cuando se desordenan en lengua materna, y que los elementos del relato como el principio y el final son más importantes en lengua extranjera que en lengua materna. La diferencia estriba en lo que hemos explicado en el punto anterior: los lectores nativos pasan menos tiempo en los bajos niveles del texto, y pueden orientar su atención a los altos niveles, como la estructura del relato, reorganizándolo. Los lectores no nativos, al ser mucho más dependientes de la codificación lingüística, realizan con mayor dificultad la reorganización estructural.

En su intento de ver la relación entre la organización textual y la comprensión de un texto en lengua extranjera, Carrell (1992), en otro estudio, propone dos textos, uno de estructura comparación-contraste, y otro, una descripción. No se comprende significativamente mejor un tipo de texto u otro. Los estudiantes que siguen el mismo esquema organizativo en sus resúmenes que en el texto leído son los que comprenden mejor los dos tipos de texto. Esto significa que los que mejor comprenden el texto hacen uso de la estructura textual para comprender el texto. Por lo tanto, ser conscientes de la estructura textual y utilizarla para comprender redunda en una mayor obtención de información.

En lo relativo al Francés Lengua Extranjera, destacan los estudios que mencionamos a continuación.

En un experimento de López Alonso y Séré de Olmos (1996) se propone a estudiantes con muy pocos conocimientos de francés, la comprensión de tres tipos de texto: narrativo (un suceso), expositivo (una receta de cocina) y argumentativo (una publicidad de Carte Orange). Los dos primeros textos no plantearon problemas de comprensión, pero sí el tercero, ya que los estudiantes no pudieron captar el tipo de texto de que se trataba, lo que bloqueó la elaboración del significado. En este caso, tipo de texto se asocia también con la referencia cultural; al ser probablemente desconocida para muchos, los estudiantes no pudieron identificar el tipo de texto.

Souchon (2000), especialista francés en comprensión lectora, aborda la competencia textual desde el punto de vista de la comprensión. Parece que el lector, al enfrentarse a un texto, tiende a asociarlo a una categoría textual o a un género. Se presentan 14 textos, de los cuales, 10 son literarios ( 5 narraciones, 3 descripciones, 1 argumentación, 1 poema), y 4 artículos de periódico. Los alumnos debían subrayar los pasajes que plantean dificultad de comprensión, y éstos se analizan de acuerdo con los porcentajes de palabras gramaticales (sustantivos, adjeti- 
vos y verbos), que posee cada tipo de texto. Aparecen regularidades en las mismas categorías de textos, lo que muestra que la categoría del texto influye profundamente en los procesos de lectura, hasta el punto de actuar como una guía de lectura.

Arévalo (2005) propone a 121 estudiantes de Francés Lengua Extranjera de cuatro niveles de la Escuela Oficial de Idiomas -desde $2^{\circ}$ a $5^{\circ}$-, un texto argumentativo, en 4 variantes:

1. Estructura argumentativa original. Título original

2. Estructura argumentativa original. Título modificado

3. Estructura argumentativa alterada. Simplificación lingüística. Título original.

4. Estructura argumentativa alterada. Simplificación lingüística. Título modificado

Los estudiantes debían realizar un resumen del texto original. Se analizan los resúmenes de acuerdo con los errores y la aparición de marcas de argumentatividad.

Algunos de los resultados muestran que:

- el esquema argumentativo ordenado es el que mejor se comprende, siendo esta variable de mayor incidencia para la comprensión que el nivel lingüístico del texto.

- los lectores del esquema argumentativo alterado tienden a reorganizar ordenadamente el texto en sus resúmenes, lo que muestra que existe un esquema argumentativo en la comprensión lectora. $\mathrm{O}$ dicho de otra manera, comprender un texto argumentativo pasa por el reconocimiento de dicho esquema argumentativo.

- el esquema argumentativo ordenado y coherente ayuda al lector a percatarse mejor de los niveles altos del texto, como el tipo de texto.

En la misma línea de estudios (Ruiz de Zarobe, en prensa), analizamos la comprensión de un texto argumentativo en francés lengua segunda por parte de estudiantes adolescentes, a través del resumen del texto en español lengua materna. Centrándonos únicamente en lo que aquí nos ocupa, el $60 \%$ de los resúmenes reproduce la estructura del texto original, que es Argumentos + Tesis-conclusión, una estructura argumentativa de tipo inductivo; pero casi el $30 \%$ reconstruyen, en el resumen, un esquema nuevo, de tipo deductivo: Tesis-Argumentos(Conclusión), lo que indica que ese esquema argumentativo lo poseen los estudiantes de manera interiorizada, y que comprender un texto argumentativo consiste en detectar la tesis y los argumentos; la organización de la información parece resultar menos importante.

\section{CONCLUSIONES}

De todas estas investigaciones, podemos concluir:

1. Parece clara la existencia psicológica de esos esquemas textuales cognitivos, que permiten tratar la información de los textos (en lengua materna) de acuerdo con sus diferentes tipos. Esos esquemas textuales funcionan como herramientas cognitivas que permiten identificar, comprender y producir distintos tipos de texto. 
Los individuos, a lo largo de su aprendizaje, van progresivamente elaborando esos esquemas a medida que los van adquiriendo. Su adquisición se produce por impregnación cultural y por convención.

2. En lo relativo a la comprensión lectora, la tipología textual, en cuanto reconocimiento de una estructura retórica de una determinada forma, ejerce una función en la comprensión lectora, ayuda a comprender y memorizar mejor la información del texto.

Dos rasgos permiten comprender mejor:

- el orden canónico o prototípico de la estructura

- la coherencia textual

Esa organización textual canónica aligera los tratamientos cognitivos que es preciso realizar para comprender, y por tanto, facilita la comprensión. Pasar de una linearidad de informaciones a una representación mental de la situación resulta menos costoso tanto en lengua materna como en lengua extranjera, aunque en lengua extranjera resulta más costoso que en lengua materna.

Además, ser conscientes de la estructura textual y hacer uso de ella posee un efecto favorable en la comprensión y permite retener mayor información.

3. El esquema textual que se activa en la comprensión es un esquema interiorizado en un orden o de manera prototípica. Esto se comprueba porque tanto en lengua materna como en lengua extranjera, cuando el orden del esquema presentado no es el canónico, se tiende a restablecer el orden usual.

Gran parte de los trabajos de investigación en lengua extranjera manipulan la estructura canónica del tipo de texto, lo que va orientado sobre todo a la captación de la estructura.

Sin embargo, la investigación actual aún no ha aportado respuestas suficientes a algunas cuestiones, y en nuestra opinión debe progresar en dos direcciones:

a) En cuanto a la influencia del tipo de texto en la comprensión, en lengua materna, si bien el tratamiento de la tipología textual convoca distintas capacidades cognitivas, como hemos visto, no resulta claro si cada tipo de texto, en su comprensión, convoca el mismo tipo de operaciones cognitivas o no: el esquema textual, el dominio de referencia, capacidades lingüísticas, etc. ¿Todas? ¿Sólo algunas? ¿Diferentes en cada caso? ¿Cuáles son las que dan cuenta de las diferencias de tratamiento de cada tipo?

b) En cuanto a la influencia del tipo de texto en la comprensión en lengua extranjera, no existen suficientes resultados que muestren si el desencadenamiento de los procesos de alto nivel, como la captación de la estructura textual, posee mayor relevancia, para la comprensión, que la competencia lingüística, o puede activarse con cierta independencia de la competencia lingüística. Sería preciso mostrar con mayor precisión en qué pro- 
cesos cognitivos interviene la tipología textual cuando se trata de una lengua extranjera, y el rol de la lengua extranjera en ese caso.

Si atendemos a las implicaciones pedagógicas de las investigaciones mostradas, aunque resulta cierto lo que afirman Gaonac'h y Fayol (2003:31) "cet effet facilitateur de la structure conventionnelle des textes sur la compréhension n'implique pas que les enfants ou les adultes aient conscience de cette organisation ", creemos que es indudable que esa conciencia ayudaría a reconocer las organizaciones de los distintos tipos de textos, y produciría un efecto favorable en la comprensión. Sería así pues deseable exponer a los estudiantes de francés a abundantes textos de distinta tipología, sensibilizarles a su construcción, proponer actividades de análisis de estructuras de distintos tipos de texto, para adquirir la forma convencional de los textos, y activar así en los estudiantes una mejor comprensión, así como un tratamiento más completo, rápido y eficaz de la información textual.

\section{BIBLIOGRAFÍA}

Adam, J.M. (1992). Les textes, types et prototypes. Récit, description, argumentation et dialogue. Paris: Nathan.

Adam, J.M. (1999). Linguistique textuelle : Des genres de discours aux textes. Paris: Nathan.

Adam, J.M. (2005). La linguistique textuelle. Introduction à l'analyse textuelle des discours. Paris: A. Colin.

Arévalo, M.J. (2005). La comprensión lectora del texto argumentativo en Francés Lengua Extranjera. Universidad del País Vasco: Servicio Editorial, Serie Tesis Doctorales.

Blanc, N. y D., Brouillet (2005). Comprendre un texte. L'évaluation des processus cognitifs. Paris: Éd. In Press.

Brassart, D.G. (1996). "Does a prototypical argumentative schema exist? Text recall in 8 to 13 years olds". Argumentation, 10: 163-174.

Carrell, P.L. (1984). "The effects of rethorical organisation on ESL readers." Tesol Quarterly, 183: 441-465.

Carrell, P.L. (1990) "Culture et contexte dans la lecture en langue étrangère : rôle des schémas de contenu et des schémas formels", en D., Gaonac'h "Acquisition et utilisation d'une langue étrangère: l'approche cognitive", Le français dans le monde: recherches et applications, Paris: Hachette.

Carrell, P.L. (1992). "Awareness of text structure: Effects on recall." Language Learning, 42: 1-2.

Coirier, P. y alt. (1990). "Le traitement du discours argumentatif: recherches en production et en compréhénsion". Archives de psychologie, 58: 315-348.

Coirier, P. y E. Marchand (1994). "Writing argumentative texts, a typological and structural approach", en G. Eigler y alt. (eds.). Writing, Current Trends in european Research. Freiburg: Hochschul Verlag.

Coirier, P. y alt. (1996). Psycholinguistique textuelle. Approche cognitive de la compréhension et de la production des textes. Paris: A. Colin.

Denhière, G. (1984). Il était une fois... Compréhension et souvenir de récits. Lille: PUL.

Denhière, G. y alt. (1992). Lecture, compréhension de texte et science cognitive. Paris: PUF.

Fayol, M. (1985). Le récit et sa construction. Neuchâtel: Delachaux et Niestlé. 
Fayol, M. (1991). "Text typologies: a cognitive approach”, en G., Denhière, y J.P. Rossi (eds.). Texts and text processing. Amsterdam: North Holland.

Fayol, M. (1996). "Comment le lecteur comprend le récit". Revue des Sciences Humaines, 60: 20- 22.

Fayol, M. (2000). "Comprendre et produire des textes écrits. L'exemple du récit”, en M., Kail y alt. (eds.). L'acquisition du langage 2. Paris: PUF: 183-213.

Gaonac'h, D. (2000). "La lecture en LE: un tour d'horizon d'une problematique de psychologie cognitive". AILE, 13: 5-14.

Gaonac'h, D. y M. Fayol (2003). Aider les élèves à comprendre: du texte au multimédia. Paris: Hachette.

Golder, C. y P. Coirier (1994). "Argumentative text writing, developmental trends”. Discourse Processes, 18: 187-210.

Golder, C. y P. Coirier (1996). "The production and recognition of typological argumentative text markers". Argumentation, 10: 271-282.

Golder, C. y D. Gaonac'h (1998). Lire et comprendre: Psychologie de la lecture. Paris: Hachette.

Kintsch, W. y T. Van Dijk (1983). Strategies of discourse comprehension. New York: Academic Press.

López Alonso, C. y Séré de Olmos (1996). "Typologie des textes et stratégies de la compréhension en LE". Études de Linguistique Appliquée, 104: 441-450.

Meyer, B.J.F. (1975). The organisation of prose and its effects on memory. Amsterdam: North Holland.

Ruiz de Zarobe, L. (2005). "Modification syntaxique de l' « input » et compréhension écrite du FLE". Travaux de didactique du FLE, 54: 71-92.

Ruiz de Zarobe, L. (2006) "The effects of the argumentative text type in the understanding of French as a foreign language", Comunicación presentada al 16th Annual Conference of the European Second Language Association (EUROSLA 2006). Turquía.

Ruiz de Zarobe, L. (2007). "Comprendre un texte descriptif en français langue étrangère ", Comunicación presentada al Symposium 2007 of the Association for French Language Studies. Francia.

Ruiz de Zarobe, L. (en prensa). "La compréhension écrite du texte argumentatif en français langue seconde".

Souchon, M. (2000). "Lecture de textes en langue étrangère et compétence textuelle". AILE 13: $15-40$. 